\title{
Screening of Helicobacter Pylori Infection in the Health Examination Detected by Urea Breath Test and Barium Meal Study
}

\author{
Fumihiro Yamasawa ${ }^{1}$, Yuichi ICHINOSE ${ }^{2}$, Emiko KANAI ${ }^{2}$, Makoto YoneMARU $^{2}$, \\ Hitoshi IsHII $^{3}$ and Keisuke ToYAMA ${ }^{2}$ \\ ${ }^{1}$ Marubeni Clinic, ${ }^{2}$ First Department of Internal Medicine, Tokyo Medical College and ${ }^{3}$ Westron Co.
}

\begin{abstract}
Screening of Helicobacter Pylori Infection in the Health Examination Detected by Urea Breath Test and Barium Meal Study: Fumihiro Yamasawa, et al. Marubeni Clinic-Background: Helicobacter pylori, $H$. pylori, is now assumed to be a definite cause of gastric cancer. Health examinations for the early detection of atherosclerotic diseases and malignant diseases have been widely undertaken in Japan, where the incidence of gastric cancer is still one of the highest in the world. To investigate $H$. pylori infection non-invasively in the regular health examinations, we introduced the urea breath test (UBT) with a barium meal study in the ordinary system. Methods: Sixty male subjects were studied (mean age: $47.8 \mathrm{y} / 0$ ) in this study. After blood examination for anti $\mathrm{H}$. pylori lgG antibody, the reference expired gas was collected from each subject. Then the subject took $100 \mathrm{mg}$ of ${ }^{13} \mathrm{C}$-urea dissolved in water with tartaric acid and sodium bicarbonate, which was used to distend the stomach, and an ordinary barium meal study was undertaken. Several rotations of the body were added to subjects for fluoroscopy. Thirty minutes after ${ }^{13} \mathrm{C}$-urea intake, the expired gas was collected again as the second gas sample. The ratio of ${ }^{13} \mathrm{C}$ to ${ }^{12} \mathrm{C}$ in the reference and second expired gas sample was measured with a mass spectrometer and the changed fraction of the isotope concentration, $d$, was calculated in each subject. The $X$-ray films of the barium meal study were read independently by one roentgenologist who was not informed at all about this study. Cases were divided into 2 groups: one group without mucosal inflammatory diseases and another group with mucosal inflammatory diseases. Results: The d value for subjects with positive $H$. pylori antibody and with mucosal inflammatory diseases, who were strongly suggestive of positive $H$. pylori infection, had a significantly higher value (d: $2.492 \pm 1.372, p<0.001$ )
\end{abstract}

Received Dec 9, 1996; Accepted June 10, 1997

Correspondence to: F. Yamasawa, Marubeni Clinic, 4-2 Ohtemachi 1, Chiyoda, Tokyo 100-88, Japan than that for subjects with negative $H$. pylori antibody and without mucosal inflammatory diseases, who were thought not to have $\mathrm{H}$. pylori infection (d: $0.141 \pm 0.107)$. Subjects who were strongly suggestive of $\mathrm{H}$. pylori infection showed distinctly higher value. Conclusions: Barium did not interfere with the reaction of $\mathrm{H}$. pylori and ${ }^{13} \mathrm{C}$-urea in the gastric mucosa. UBT can be performed non-invasively together with a barium meal study and it provides useful information on $\mathrm{H}$. pylori infection in the health examination.

(J Occup Health 1998; 40: 32-36)

Key words: Helicobacter pylori, Urea breath test, Barium meal study, Stable isotope, Mass spectrometer, Anti Helicobacter pylori IgG, Gastric cancer, Health examination

Health examinations for the early detection of atherosclerotic diseases and malignant diseases have been widely undertaken in Japan, where the incidence of gastric cancer is still extremely high. Barium meal studies to detect stomach cancer are commonly used for the health examination. Since the report from WHO in $1994^{1)}$ identifying Helicobacter pylori ( $H$. pylori) as a definite cause of stomach cancer, it has become very important to diagnose $H$. pylori infection efficiently and noninvasively in health examinations. Though it is widely accepted that barium meal studies have contributed to the detection of stomach cancer, they cannot detect the $H$. pylori infection. Recently, several examinations for the detection of $H$. pylori infection have been introduced, such as the mucosal biopsy method, culture method ${ }^{2)}$, rapid urease test ${ }^{3)}$, polymerase chain reaction method ${ }^{4)}$, serum anti $H$. pylori IgG test ${ }^{5)}$ and urea breath test (UBT) ${ }^{6}$. These first 4 tests can be conducted with the biopsy specimen obtained at the gastrofiberscopic examination, which is an invasive method. Serum anti $\mathbf{H}$. 
pylori IgG test is a non-invasive screening test but positive $\mathrm{H}$. pylori IgG suggests not only present but also past $H$. pylori infection. Positive $H$. pylori IgG does not mean definite present $H$. pylori infection. In contrast to these other examinations, UBT is a non-invasive and simple procedure, which is based on the collection of expired gases before and after urea intake. With a mass spectrometer and personal computer, the results can be obtained simultaneously. If UBT is positive, it strongly suggests that the subject presently has $H$. pylori infection. In the ordinary health examinations, because several medical tests need to be performed efficiently, simply and non-invasively, it would be useful to introduce UBT in combination with a barium meal study for the detection of $\mathrm{H}$. pylori infection. We studied the possibility of the introduction of UBT in the health examination system and how the barium meal study affected the results of UBT.

\section{Methods}

Sixty men (age: $35-58$, mean: $47.8 \mathrm{y} / \mathrm{o}$ ) were selected randomly in a company-based yearly health examination and oral consent for this study was obtained from each of them. Twenty-five of these subjects had histories of gastroduodenal symptoms. Serum anti H. pylori IgG test and UBT were added to the ordinary examinations, which include urinalysis, blood test, blood pressure measurement, electrocardiography, chest $X$ ray and barium meal study. Serum anti H. pylori IgG were measured qualitatively with the pilikaplate $G$ Helicobacter EIA kit (Biomerica, USA). Results of $(-)-(+-)$ were considered negative for serum anti $\mathbf{H}$. pylori IgG and those of $(+)-(+++)$ were thought to be positive for serum anti $\mathrm{H}$. pylori IgG.

Stable isotope ${ }^{13} \mathrm{C}$-labelled urea (Isotec Inc., Dayton, Ohio, USA) and a mass spectrometer (WSMR-1400, Westron Co., Chiba, Japan) were used for the UBT test. The mass spectrometer equipped with ${ }^{12} \mathrm{CO}_{2}$ and ${ }^{13} \mathrm{CO}_{2}$ detectors could analyse ${ }^{12} \mathrm{CO}_{2}$ and ${ }^{13} \mathrm{CO}_{2}$ gases simultaneously and continuously. A reference expired gas sample (Gas 1) was obtained from each subject by collecting their expired gas from the level of their functional residual capacity into the 1 liter aluminum sampling bags. Just before the barium meal study, the subjects took $4.5 \mathrm{~g}$ of Balgin effervescent granules (Kaigen Pharma. Co., Osaka, Japan, tartaric acid and sodium bicarbonate) with $100 \mathrm{mg}$ of ${ }^{13} \mathrm{C}$ labelled urea dissolved water in order to distend the gastric inner surface for double contrast study. Subjects underwent an ordinary barium meal study with fluoroscopy (KXO-80 DAW-10A, Toshiba,
Tokyo, Japan) and ${ }^{13} \mathrm{C}$-labelled urea was diffusely distributed on the gastric inner surface by rotating their bodies. A second expired gas sample (Gas 2) was taken from each subject in the same manner 30 min after ${ }^{13} \mathrm{C}$-labelled urea intake. Subjects rinsed out their mouths sufficiently with water before each gas sampling. Expired gas was measured simultaneously and continuously with a mass spectrometer while the subjects exhaled their gases. The concentrations of ${ }^{12} \mathrm{CO}_{2}$ and ${ }^{13} \mathrm{CO}_{2}$ gases confirmed that the end-expired gas had the highest values in their expiration. Signals of reference and second expired gases in bags were transferred through $\mathrm{AD}$ converters to a personal computer (RL-600, Westron Co., Chiba, Japan) and the following data analysis was performed.

As a small amount of stable isotope ${ }^{13} \mathrm{C}$ is present both in nature and in the body, the ${ }^{13} \mathrm{CO}_{2} /{ }^{12} \mathrm{CO}_{2}$ ratio in expired gases in each subject was calculated as the changed fraction, $\mathrm{d}$, of the isotope concentration in Gas 1 and Gas 2.

$$
\begin{aligned}
& \mathrm{d}=100 \times\left({ }^{13} \mathrm{CO}_{2} /{ }^{12} \mathrm{CO}_{2} \text { in Gas } 2-{ }^{13} \mathrm{CO}_{2} /{ }^{12} \mathrm{CO}_{2}\right. \\
& \text { in Gas 1) } /{ }^{13} \mathrm{CO}_{2} /{ }^{12} \mathrm{CO}_{2} \text { in Gas } 1 \\
& \text { Gas 1 : reference expired gas sample } \\
& \text { Gas 2: second expired gas sample }
\end{aligned}
$$

The $d$ values were obtained from 6 subjects who took only $4.5 \mathrm{~g}$ of effervescent granules without any urea (granules group). The ${ }^{13} \mathrm{C} /{ }^{12} \mathrm{C}$ ratio of $\mathrm{CO}_{2}$ gas which were effervesced after adding $\mathrm{HCl}$ solution to effervescent granules was also measured.

The roentgen films of barium meal study were read independently by one roentgen specialist who knew nothing about this study. The roentgenologic findings were divided into 2 groups: one group without mucosal inflammatory diseases (normal findings, gastric polyps, duodenal polyps, gastric submucosal tumor and esophageal hiatal hernia) and the other group with mucosal inflammatory diseases (chronic gastritis, erosive gastritis, gastric ulcer scar, duodenal ulcer scar).

Results were expressed as means \pm SD and/or their ranges. Students' t-test or one way analysis of variance (ANOVA) was performed, and when allowance was made for the $F$ value, the significance between groups was computed by using the Scheffe test. Probability values of $<0.05$ were considered significant.

\section{Results}

\section{1) Anti H. pylori IgG antibody and barium meal study}

Twenty-six cases (43\%) showed negative results and the other 34 cases ( $57 \%$ ) were positive for anti H. pylori IgG antibody. There were 26 cases (43\%) without inflammatory diseases and 34 cases (57\%) 
Table 1. Classiflcation of subjects

\begin{tabular}{|c|c|c|}
\hline $\begin{array}{r}\text { Ba Meal } \\
\text { study }\end{array}$ & without inflammation & with inflammation \\
\hline Negative & 20 (group 1 ) & 6 (group 2) \\
\hline Positive & 6 (group 3 ) & 28 (group 4) \\
\hline
\end{tabular}

IgG: serum anti H. Pylori IgG. Each number: number of subjects in the group.

with inflammatory diseases according to the barium meal study. Subjects were classified into 4 groups (Table 1); group 1 consisted of cases with negative IgG and without inflammation, group 2 consisted of cases with negative $\operatorname{IgG}$ and with inflammation, group 3 consisted of cases with positive IgG and without inflammation, and group 4 consisted of cases with positive $\mathrm{IgG}$ and with inflammation. There were 20 cases in group 1,6 in group 2,6 in group 3 and 28 in group 4.

\section{2) The ${ }^{13} \mathrm{C} /{ }^{12} \mathrm{C}$ of $\mathrm{CO}_{2}$ gas of effervescent granules}

The ratio of ${ }^{13} \mathrm{CO}_{2}$ to ${ }^{12} \mathrm{CO}_{2}$ of effervescent granules was 0.01 , which was the same value as reported in the literature?

\section{3) The d values}

The $\mathrm{d}$ value in the granules group was $0.017 \pm$ 0.089 ( $-0.114-0.126)$. Subjects with negative $H$. pylori $\operatorname{IgG}$ had $0.438 \pm 0.766(-0.132-3.503)$ and subjects with positive $H$. pylori IgG had $2.243 \pm$ $1.313(0.097-6.802)$ in $d$ value, which was significantly higher than that for negative $\mathrm{H}$. pylori IgG ( $p<0.0001$, Fig. 1). The $d$ values were $0.141 \pm$ $0.107(-0.132-0.340)$ in group $1,1.429 \pm 1.164$ (0.17-3.503, not significant compared to group 1) in group $2,2.102 \pm 1.021(0.848-3.140, \mathrm{p}<0.01)$ in group 3 and $2.492 \pm 1.372(0.097-6.802, p<0.001)$ in group 4 . The $d$ value was the lowest in group 1

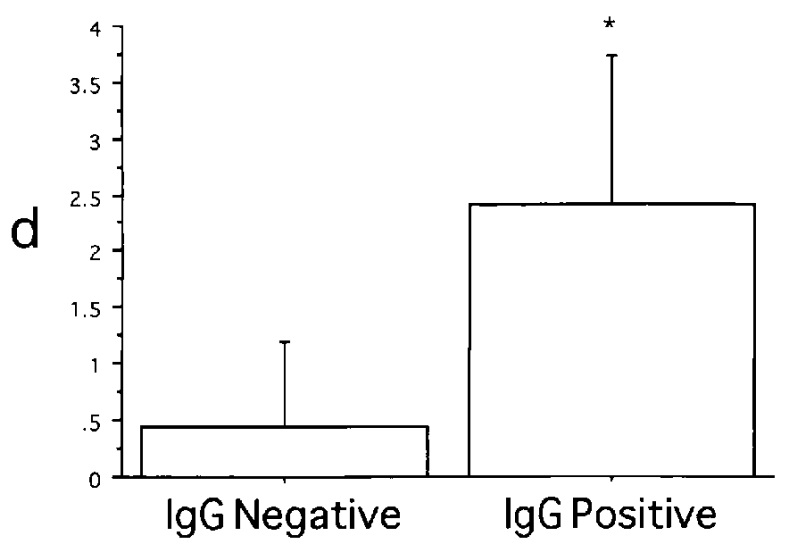

Fig. 1. Subjects with positive Helicobacter pylori IgG had significantly higher $d$ values than those with negative IgG. $\star$ : $\mathbf{p}<0.0001$ compared to IgG negative subjects.

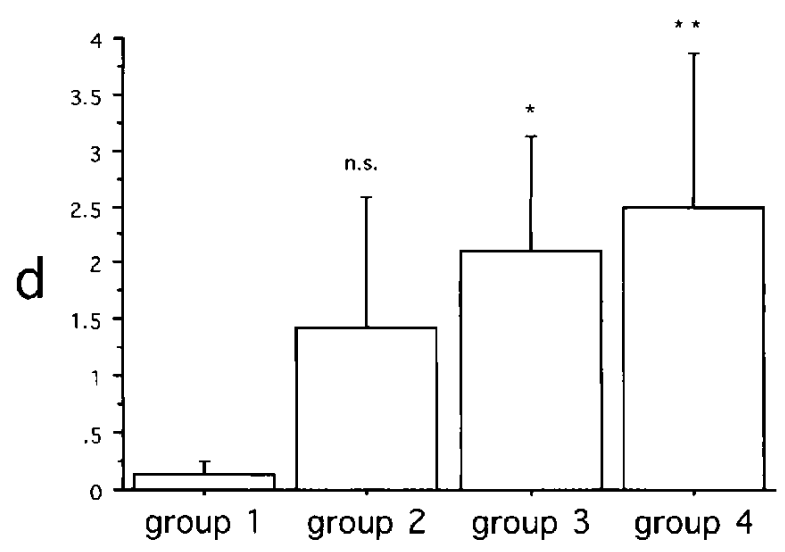

Fig. 2. Groups 3 and 4 had significantly higher $d$ values than that of group $1 .^{\star}: \mathrm{p}<0.01$, compared to group $1 .^{\star \star}: \mathrm{p}<$ 0.001 , compared to group 1. n.s.: not significant, compared to group 1.

and the highest in group 4 (Fig. 2). As the d value in the granules group was negligibly small, it could be assumed that $\mathrm{CO}_{2}$ gas in effervescent granules did not alter other $d$ values.

\section{Discussion}

Since Warren and Marshall found the bacterium $^{8)}$, now called Helicobacter pylori in the stomach in 1987, many data have been published relating it to gastroduodenal diseases. Among them, a report ${ }^{1)}$ from the National Institutes of Health Consensus Conference in 1993 which declared infection with $\mathbf{H}$. pylori to be an important cause of duodenal and gastric ulcers and a report ${ }^{9}$ from the International Agency for Research on Cancer in 1994 which classified $H$. pylori as a group 1 carcinogen, a definite cause of gastric adenocarcinoma in humans, posed the question of how infection with H. pylori could be diagnosed efficiently and noninvasively in the health examinations which have come to be widely accepted in Japan. Non-invasive barium meal studies have been included in the health examinations for screening of gastric and duodenal diseases. When needed, the gastrofiberscopic examination should also be performed to detect infection with $\mathrm{H}$. pylori. Microscopic examinations, bacterial culture method ${ }^{2)}$, rapid urease test ${ }^{3)}$ and PCR test ${ }^{4)}$ for detection of the infection should follow with biopsy tissue specimens. Although the gastrofiberscopic examination is a one type of gold standard for detection of the infection, it is not a non-invasive method for screening and it could unfortunately give false negative results with regard to infection. The importance of anti $\mathrm{H}$. pylori IgG an-

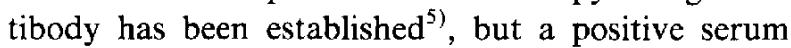
antibody can mean both present and past $\mathrm{H}$. pylori infection. It was also reported ${ }^{5)}$ that the sensitivity 
and specificity for the $\mathrm{H}$. pylori infection detected by anti $\mathrm{H}$. pylori IgG antibody were $94.2 \%$ and $75.6 \%$, respectively.

In contrast to other examinations, UBT is a noninvasive method and can reveal the presence of urease activity in gastric mucosa. Urease lyzes one urea molecule into 2 ammonia molecules and one $\mathrm{CO}_{2}$ molecule. The carbon dioxide molecule is easily absorbed into capillaries in the gastric mucosal surface and then excreted from the lungs. As a carbon atom in the urea molecule is labelled with stable isotope, ${ }^{13} \mathrm{C}$ or ${ }^{14} \mathrm{C}$, it can easily be detected in expired gas with a mass spectrometer. H. pylori has a strong urease activity. It can neutralize its environment with ammonia by urease activity even in the presence of highly acidic gastric juice. As urease activity cannot be detected in the normal gastric mucosa, positive UBT means the presence of $\mathbf{H}$. pylori in gastric mucosa. The results of UBT are not affected by the uneven distribution of $\mathrm{H}$. pylori over the gastric surface, which occasionally gives false negative results with gastric biopsy methods. Both sensitivity and specificity were reported to be more than $95 \%$ in $\mathrm{UBT}^{11)}$. It could be thought that UBT is more accurate than the serum antibody method. Some bacteria, such as anaerobes in the oral cavity and Helicobacter species, are known to have urease activity. Simultaneous and continuous expired gas measurements were able to be performed with a mass spectrometer, which showed that the concentrations of ${ }^{13} \mathrm{CO}_{2}$ and ${ }^{12} \mathrm{CO}_{2}$ were the highest in the end expired phase. This finding means that ${ }^{13} \mathrm{CO}_{2}$ was derived not from the mouths but from the lungs. ${ }^{13} \mathrm{CO}_{2}$ could therefore be thought to be derived from the stomach. The infrared $\mathrm{CO}_{2}$ spectrometer has been introduced in $\mathrm{UBT}^{10)}$, but for continuous monitoring of the $\mathrm{CO}_{2}$ concentration in expired gas, the mass spectrometer is much preferred. UBT is non-invasive and can be introduced into a routine test in the health examination.

Since Graham reported UBT in $1987^{6)}$, clinical applications have been tried extensively ${ }^{10}$. There still has been no report on an efficient combination of UBT with a barium meal study for screening of infection with $\mathrm{H}$. pylori. If the combination study works efficiently for the detection of $\mathbf{H}$. pylori, prevention and detection of gastric cancer and gastroduodenal ulcer will become simplified. In this study, $100 \mathrm{mg}$ of ${ }^{13} \mathrm{C}$-labelled urea was dissolved in water with which subjects swallow effervescent granules to distend the gastric inner surface for a double contrast study. In contrast to the ordinary UBT, many rotations of body were added for fluoroscopic examinations and the ${ }^{13} \mathrm{C}$-labelled urea was diffusely distributed over the gastric mucosal surface. As the ratio of ${ }^{13} \mathrm{C}$ to ${ }^{12} \mathrm{C}$ in effervescent granules was the same as that reported one in the literature, effervescent granules did not affect the UBT data. The $d$ value with anti $H$. pylori IgG positive subjects was significantly higher than that of IgG negative subjects. Barium did not interfere with the reaction of $\mathrm{H}$.pylori and ${ }^{13} \mathrm{C}$-labelled urea in the gastric mucosa because subjects with present and/or past $H$. pylori infection had the higher $d$ values.

Subjects were categorized into 4 groups: group 1 consisted of cases with negative IgG and without roentgenologically inflammatory diseases, group 2 consisted of cases with negative IgG and with roentgenologically inflammatory diseases, group 3 consisted of cases with positive IgG and without roentgenologically inflammatory diseases, and group 4 cases with positive IgG and with roentgenologically inflammatory diseases. Subjects in group 1 were thought not to have been infected with $\mathbf{H}$. pylori and those in group 4 were strongly considered to have been infected with $H$.pylori. The d value in group 4 was significantly higher than that in group 1 . There were 20 subjects in group 1 and all had a $d$ value of less than 0.5 , which is the standard value for normal non-infected subjects ${ }^{12)}$. Other groups had higher $d$ values than the standard.

Barium did not interfere with the reaction of $\mathbf{H}$. pylori and ${ }^{13} \mathrm{C}$-urea in gastric mucosa, and ${ }^{13} \mathrm{C}$-UBT with the barium meal study seemed to be an efficient, non-invasive method for screening of $\mathbf{H}$. pylori infection. It is recommended that UBT be introduced into health examinations as a routine study. Further investigations should consider the costs, labor and benefits of UBT in health examination systems.

\section{References}

1) World Health Organization. Infection with Helicobacter pylori. In: IARC monographs on the evaluation of carcinogenic risks to humans. Vol. 61. Schistosomes, liver flukes and Helicobacter pylori. Lyon, France: International Agency for Research on Cancer, 1994: 177-240.

2) Ohkura T, Okayasu I, Yamada M, et al. A high frequency of detection of Helicobacter pylori in whitish exudate of gastric ulcer. J Clin Gastroenterol 1991; 13: 649-655.

3) Marshall BJ, Warren R, Francis GJ, et al. Rapid urease test in the management of Campylobacter pyloridis-associated gastritis. Am J Gastroenterol 1987; 82: 200-210.

4) Clayton CL, Kleanthous $H$, Coates PJ, et al. Sensitive detection of Helicobacter pylori by using poly- 
merase chain reaction. J Clin Microbiol 1992; 30: 192-200.

5) Fukuda Y, Yamamoto $K$, Mizuta $T$, et al. Evaluation of a commercial kit, Pilikaplate G Helicobacter, for diagnosis of Helicobacter pylori infection. Medicine and Pharmacy 1994; 31: 321-328.

6) Graham DY, Klein PD, Evans DJ, et al. Campylobacter pylori detected noninvasively by the ${ }^{13} \mathrm{C}$ urea test. Lancet 1987 ; i : 1174-1177.

7) Science table: Physics/Chemistry, atom. molecule. nucleus. stable isotope, Physics 132 (552), National Institute of Astrology, 69ed., 1996.

8) Warren JR, Marshall BJ. Unidentified curved bacilli on gastric epithelium in active chronic gastritis. Lancet 1983; i : 1273-1275.
9) NIH Consensus Development Panel on Helicobacter pylori in Peptic Ulcer Disease. Helicobacter pylori in peptic ulcer disease. JAMA 1994; 272: 65-69.

10) Ohara H, Suzuki T, Nakagawa $T$, et al. ${ }^{13} \mathrm{C}-\mathrm{UBT}$ using an infrared spectrometer for detection of Helicobacter pylori and for monotoring the effect of Lansoprazole. J Clin Gastroenterol 1995; 20 (Suppl. 2) : S115-S117.

11) Miwa $H$, Sato $N$. Non-invasive diagnosis for $H$. pylori infection. J Clin Experi Med (Igaku no Ayumi) 1996; 179: 69-72.

12) Logan RPH, Dill S, Bauer FE, et al. The European ${ }^{13} \mathrm{C}$-urea breath test for the detection of Helicobacter pylori. Eur J Gastroenterol Hepatol 1991; 3: 915921. 